\title{
Targeted Genome Sequencing
}

National Cancer Institute

\section{Source}

National Cancer Institute. Targeted Genome Sequencing. NCI Thesaurus. Code C158253.

A DNA sequencing technique in which selected regions of an individual's genome are sequenced. 